\title{
The Transmissible Nature of the Genetic Factor in Escherichia coli that Controls Haemolysin Production
}

\author{
By H. WILLIAMS SMITH AND SHEILA HALLS \\ The Animal Health Trust, Stock, Essex
}

(Accepted for publication 28 November 1966)

\begin{abstract}
SUMMARY
In mixed cultures, a genetic factor (designated Hly factor) responsible for $\alpha$-haemolysin production in 10 of 53 strains of Escherichia coli was transmitted at a relatively high rate to other organisms, including shigellas and salmonellas as well as $E$. coli. Transmission was evidently by conjugation for it was not achieved by bacteria-free culture fluids. Although the factor was not eliminated by acriflavine or ultraviolet irradiation, it was probably a plasmid. Salmonella recipients were very unstable, and during serial subculture in broth the Hly factor was lost from most of these organisms. The factor was easily reintroduced into these segregants, but with difficulty or not a) all into the rare $E$. coli organisms which had lost it. Hly factor was transmitted independently of F, R and col factors and phages were not involved in its transmission. Strains of $E$. coli $\mathrm{K} 12 \mathrm{~F}^{+}$into which Hly factor had been introduced became resistant to the F-specific phage. From this it appeared that the factor has the $f^{+}$character observed in certain $\mathrm{R}$ factors. The illness produced in mice by the intravenous injection of culture fluids of $\alpha$-haemolytic strains of $E$. coli was shown to be caused by the $\alpha$-haemolysin itself.
\end{abstract}

\section{INTRODUCTION}

Smith (1963) showed that two haemolysins may be produced by Escherichia coli. The most common one, designated $\alpha$, can be obtained free from the bacteria. Strains of $E$. coli which produce it are frequently found in small numbers in the faeces of human beings, cattle, sheep and pigs. Some of these strains cause clinical disease in the pig. Certain bacterial characters-for instance, drug-resistance (Watanabe, 1963) and colicine production (Fredericq, 1963)-are controlled by genetic elements which are transmissable by conjugation between organisms of the family Enterobacteriaceae, independently of the chromosome. The present paper describes experiments in which it was shown that $\alpha$-haemolysin production was another character which could be transferred by conjugation. Ten of 53 naturally-occurring $\alpha$-haemolytic strains of $E$. coli transmitted their haemolytic activity to other bacteria, including strains of the same species, as well as to Salmonella and Shigella strains. The term Hly factor is proposed for the element which is responsible for transmissible $\alpha$-haemolysin production.

When the Hly factor was transferred to $E$. coli $\mathrm{K} 12 \mathrm{~F}^{+}$, the culture was no longer lysed by the F-specific phage MS2. In this way, the Hly factor behaved like those $\mathrm{R}$ factors which have been designated $f^{+}$(for fertility inhibition; Watanabe, 1963) because they inhibit the functions of $F$. 


\section{METHODS}

The transfer of the ability to produce $\alpha$-haemolysin. Nutrient broth (Oxoid no. 2) in $10 \mathrm{ml}$. amounts was seeded with $0.02 \mathrm{ml}$. of a $24 \mathrm{hr}$ broth culture of a haemolytic strain of Escherichia coli and with $0.02 \mathrm{ml}$. of a similar culture of a non-haemolytic strain. On occasion, the relative sizes of the inocula were varied. In each experiment the prospective recipient non-haemolytic strain was a mutant resistant to one of the drugs ampicillin, streptomycin or nalidixic acid to which the haemolytic strain was sensitive. The mixed cultures were incubated at $37^{\circ}$ for $24 \mathrm{hr}$ and then inoculated so as to yield as many as possible well-isolated colonies on 'washed blood' agar containing 20-30 $\mu \mathrm{g}$. drug $/ \mathrm{ml}$. The plates were incubated at $37^{\circ}$ for $24 \mathrm{hr}$ and then examined for haemolytic colonies. When any were present, one or two were picked and purified by plating twice on 'washed blood' agar and then examined serologically to confirm that they were colonies of the recipient strain. Auxotrophic mutants of $E$. coli strain $\mathrm{K} 12$ were used as recipients in some experiments; here, the bacteria were identified by their growth requirements. In most cases the final culture was examined to determine that the haemolysis produced was due to $\alpha$-haemolysin (Smith, 1963). In all mixed culture experiments, controls, consisting of nutrient broth that had been inoculated with the recipient strain only, were also tested.

The $\mathrm{fi}^{+}$character of Hly factors. This was tested after transfer to $\mathrm{F}^{+}$strains of Escherichia coli $\mathrm{K} 12$, by examining the cultures for visible lysis by phage MS2 (Davis, Strauss \& Sinsheimer, 1961). This has been shown to be a valid test for the $f i$ character (Meynell \& Datta, 1966).

'Washed blood' agar. This consisted of nutrient agar containing washed ox red cells in a concentration equivalent to that in $10 \%(v / v)$ ox blood.

The identification of $\alpha$-haemolysin-producing strains, the titration of cell-free $\alpha$-haemolysin, the preparation of antisera to haemolysin and the performance of plate antihaemolysin tests were done by the methods of Smith (1963).

\section{RESULTS}

The incidence of transmissible haemolysin factor (Hly factor) amongst $\alpha$-haemolytic strains of Escherichia coli

The $\alpha$-haemolytic activity of 53 epidemiologically unrelated strains of Escherichia coli belonging to 11 different serotypes and pathogenic for pigs was tested for transmissibility. In 10 of the 53 strains the haemolysin was found to be transmissible; despite repeated tests the $\alpha$-haemolysin of the remaining 43 strains was never shown to be so. Of the 10 positive strains, 7 possessed the antigenic formula O138:K81 and one the antigenic formula $0147: \mathrm{K} 89,88 \mathrm{ac}$. The other two strains belonged to the Abbotstown type, OA1:KA1,88ac, a type that has recently been found by $\mathrm{Mr}$ E. J. Sweeney and others (unpublished) to be incriminated in some outbreaks of diarrhoea in pigs; this type has not yet been given an internationally accepted formula. Of the $43 \alpha$-haemolytic strains which did not transmit their haemolytic activity 8 were of the antigenic type $0138: \mathrm{K} 81,2$ of the type $0147: \mathrm{K} 89,88 \mathrm{ac}$ and 13 of the Abbotstown type.

Most of the non-haemolytic strains used in the mixed culture experiments were able to receive the Hly factor from a donor strain. For example, the Hly factor from 
one donor strain, P 233, of the Abbotstown type, was transmitted to 15 of 18 nonhaemolytic strains of various antigenic formulae; the remaining 3 strains did not act as recipients for any of the Hly factors tested.

Although variation in the rate of transmission was sometimes noted, from donor to donor and from recipient to recipient, the rate was usually high. Frequently $10-20 \%$ of the colonies of the recipient strain which grew on the drug-containing washed blood agar plate inoculated with the $24 \mathrm{hr}$ mixed culture of recipient +donor strain were haemolytic. A typical result of such an experiment is illustrated in Pl. 1, fig. 1.

The Hly factor was transmitted at the same rate to three different variants of Escherichia coli $\mathrm{K} 12$, irrespective of whether they were $\mathrm{F}^{+}$or $\mathrm{F}^{-}$. The transfer rate was in the region of $10-20 \%$.

The presence or absence of $R$ factors (Watanabe, 1963) did not influence the ability of a strain to act as a donor or as a recipient for Hly factor; 5 of the 10 wild strains from which haemolytic activity was transmitted, and 6 of the 43 strains from which it was not transmitted, contained $\mathrm{R}$ factors. The presence or absence of Hly factors did not interfere with the ability of a strain to act as a donor or recipient of $\mathbf{R}$ factors. In 21 mixed-culture experiments, Hly factor was transmitted from one or other of 6 Hly factor-containing and $\mathrm{R}$ factor-containing strains to one or other of 16 recipient strains. Although these experiments were designed to detect transmission of Hly factor, it was noted that $R$ factors also had been transmitted in four of these experiments. Strain P 233, which contained both the Hly factor and an R factor which conferred neomycin-resistance, was grown with three different non-haemolytic neomycin-sensitive $\mathrm{K} 12$ strains, two $\mathrm{F}^{+}$and one $\mathrm{F}^{-}$. The results with all three mixtures were similar. Approximately $10 \%$ of the $\mathrm{K} 12$ organisms were now haemolytic. The proportion of neomycin-resistant organisms was much lower, about $0.01 \%$, about half of which were haemolytic. The $\mathrm{F}^{+}$strains into which neomycin resistance only had been transmitted retained their sensitivity to F-specific phage. The $\mathbf{R}$ factor was, therefore, an $f^{-}$factor. However, when haemolytic activity only or haemolytic activity + neomycin resistance had been transmitted, the $\mathrm{F}^{+}$cultures became resistant to $F$ phage. Resistance to $F$ phage was a feature of all $\mathrm{F}^{+}$strains into which Hly had been introduced. During one experiment in which Hly factor was transmitted from strain $\mathrm{P} 233$ to strain $\mathrm{K} 12 \mathrm{~F}^{+}$, about $15 \%$ of the haemolytic colonies had also acquired the $\mathrm{K} 88$ antigen from strain $\mathrm{P} 233$, thus confirming the observation of Ørskov \& Ørskov (1966) that the factor which controls this K88 antigen is transmissible in mixed culture.

\section{The transmission of Hly factor from Escherichia coli to other bacterial species}

By using several different donors, attempts were made to transmit Hly factor from Escherichia coli to four strains of Shigella sonnei and to one strain each of Shigella flexneri 2a, 3a and 4a, Salmonella typhimurium, S. dublin, S. cholerae-suis, Actinobacillus lignieresi, Chromobacterium violaceum, Klebsiella aerogenes, $K$. pneumoniae, Pasteurella pseudotuberculosis, P. septica, Proteus morgani, Pseudomonas aeruginosa, a Bacillus sp., Staphylococcus aureus and Streptococcus faecalis. Transmission was achieved to 3 of the 4 Shigella sonnei strains, the 3 Shigella flexneri strains and the 3 Salmonella strains. Despite repeated attempts it was impossible to transmit Hly factor to any of the other bacteria. The transfer rate of Hly factor to the Salmonella 
and Shigella strains $(0 \cdot 5-5 \%)$ was usually lower than that observed when $E$. coli strains were used as recipients. The Hly factor was easily retransmitted from these Salmonella and Shigella strains to non-haemolytic strains of $E$. coli.

The nature of the haemolysin produced by $\mathrm{Hly}^{+}$ and other strains of Escherichia coli

Since only 10 of $53 \propto$-haemolytic strains of Escherichia coli were shown to transmit their haemolytic activity, antisera were prepared in rabbits against the haemolysins produced by a transmitting and a non-transmitting strain. Plate anti-haemolysin tests were then made against six wild $\mathrm{Hly}^{+}$strains and six wild strains whose haemolytic activity was not transmitted. The results did not reveal any difference between the haemolysin of the two groups.

Very little difference was noted in yield of $\alpha$-haemolysin between wild strains of Escherichia coli whose haemolytic activity was not transmitted and wild strains shown to contain Hly factor, and strains of $E$. coli, Salmonella and Shigella into which Hly factor had been introduced in the laboratory.

\section{The significance of culture filtrates, phages and colicines in Hly factor mediation}

Culture filtrates. When the $\alpha$-haemolytic strain P233 was grown in mixed culture with one or other of two different non-haemolytic strains of Escherichia coli, approximately $10 \%$ of the organisms of these two strains accepted the Hly factor of strain P233. When the experiments were repeated with $6 \mathrm{ml}$. of a filtrate (which had been passed through a membrane of average pore diameter $250 \mathrm{~m} \mu$ ) of a broth culture of strain P233 in place of living organisms, no haemolytic organisms were isolated.

Phages. None of $16 \mathrm{Hly}^{+}$strains of Escherichia coli, comprising 6 naturally occurring and 10 to which Hly factors had been transferred in the laboratory, could be shown to be lysogenic when tested against 9 strains of $E$. coli and one strain each of Salmonella dublin, S. choleraesuis and Shigella sonnei, strains that had been shown to act as recipients of Hly factor.

Colicines. The two groups of strains used in the previous experiment were also examined to see whether any of the Hly-factor donors produced a colicine. Only 2 of the 16 donors were found to be colicinogenic, the colicines produced by both being active against the same 5 strains of Escherichia coli.

In some mixed cultures Hly factor was transmitted from a strain that produced colicine active against the recipient strain; in such instances the colicinogeny was not transmitted at the same time as the Hly factor. In some, but not all, cases in which a non-haemolytic strain of Escherichia coli did not act as a recipient of Hly factor this failure appeared to be associated with the fact that the strain produced a powerful colicine active against the $\mathrm{Hly}^{+}$donor itself.

\section{The stability of the Hly factor in different bacteria}

Strains known to contain Hly factor were passaged daily for 20 days in nutrient broth at $37^{\circ}$ by using small inocula transferred with the tip of a platinum wire. After incubation, each subculture was inoculated on washed blood agar to determine whether it contained any organisms that had lost the Hly factor.

Escherichia coli. The Hly factors were relatively stable in this species, for Hly- 
segregant colonies were only occasionally seen, and did not become more numerous in the later subcultures. For instance, of six wild $\mathrm{Hly}^{+}$strains, one yielded one or two non-haemolytic colonies in four subcultures and two yielded one non-haemolytic colony once; only haemolytic colonies were found with the other three strains. Seven strains into which Hly factor had been introduced in the laboratory were subcultured. Apart from one strain which yielded approximately one non-haemolytic colony to every 40 haemolytic colonies at each subculture, all the other strains consistently yielded only haemolytic colonies.

Shigella species. The three strains of Shigella sonnei into which Hly factor had been introduced in the laboratory were subcultured; one always yielded haemolytic colonies only; another gave one or two non-haemolytic organisms following a few subcultures. By contrast, the third strain was much less stable, for an increasing number of non-haemolytic colonies were found following each subculture. After the fifth subculture, the ratio of non-haemolytic to haemolytic colonies was $40: 1$. The ratioincreased to about 50:1 at the eleventh passage, and remained the same thereafter. To see whether the stability of the Hly factor in this strain would increase with time since infection, this strain was also submitted to serial subculture on washed blood agar plates, the inoculum for each subculture consisting of a suspension of a single haemolytic colony from the previous subculture. The ratio of non-haemolytic to haemolytic colonies on the washed blood agar plates fluctuated considerably but did not show any trend either towards an increase or a decrease. During the first ten subcultures it varied from $1: 1$ to $40: 1$. From the tenth to the fifteenth subculture it was approximately $30: 1$ and from the sixteenth to the twentieth approximately $200: 1$. Subculture in broth of the three Shigella flexneri strains into which Hly factors had been introduced in the laboratory resulted in the isolation of haemolytic organisms only.

Salmonella species. Instability of the Hly factor was a pronounced feature of Salmonella typhimurium, $S$. dublin and $S$. choleraesuis, into which it had been introduced in the laboratory. After each subculture in broth an increasing number of non-haemolytic organisms were isolated. By the fifth subculture the cultures appeared to consist only of non-haemolytic organisms.

By the technique used in studying the unstable strain of Shigella sonnei, the three haemolytic Salmonella strains were submitted to serial subculture on washed blood agar. The relative numbers of non-haemolytic and haemolytic colonies fluctuated considerably from subculture to subculture. In these studies the strain of Salmonella dublin was the most stable, the ratio of non-haemolytic to haemolytic colonies isolated varying from $2: 1$ to $1: 10$, usually the latter. The ratio at each of the 20 examinations in the case of the Salmonella typhimurium and $S$. choleraesuis strains ranged from $20: 1$ to $1: 10$, the latter ratio being more commonly found towards the end of the subcultivation period. However, when a single haemolytic colony from the twentieth serial subculture of both the $S$. typhimurium and $S$. choleraesuis strains was submitted to subcultivation in broth, all the colonies which grew after the third passage were non-haemolytic. The results of inoculating a washed blood agar plate with a suspension of a haemolytic colony of S. typhimurium, S. dublin, Shigella flexneri and the unstable S. sonnei strain is illustrated in P1. 1, fig. 2. 


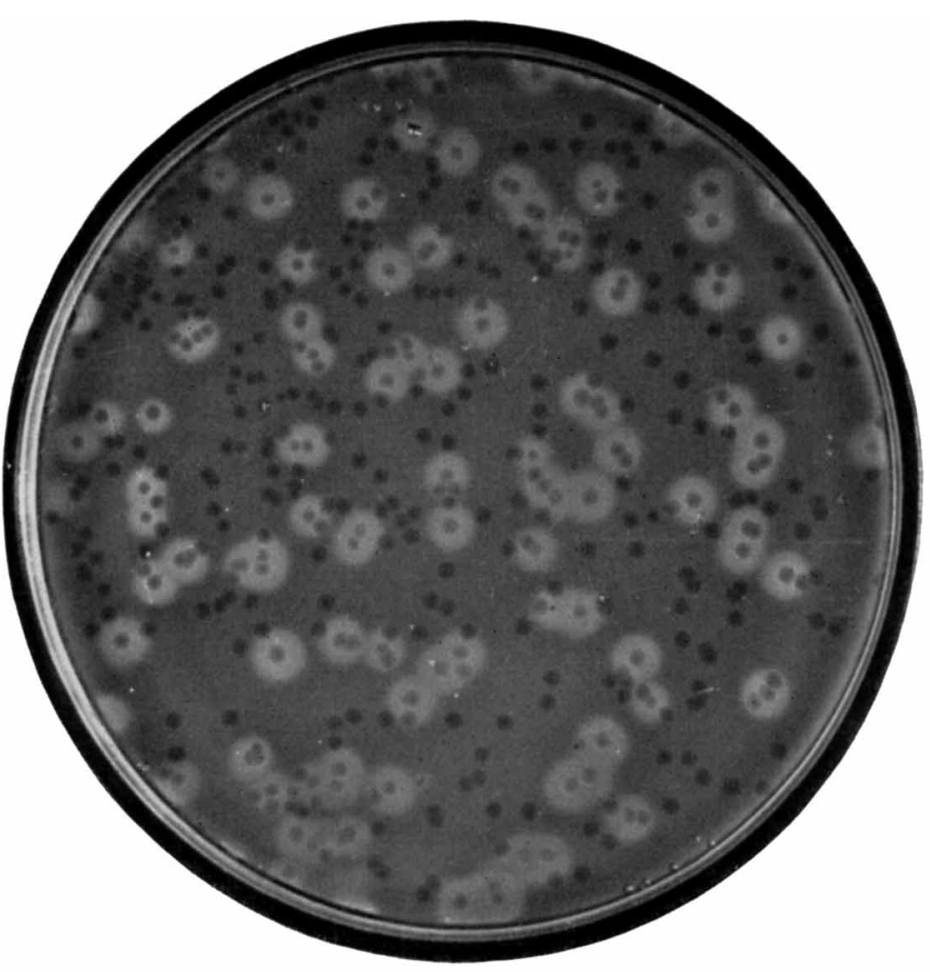

Fig. 1

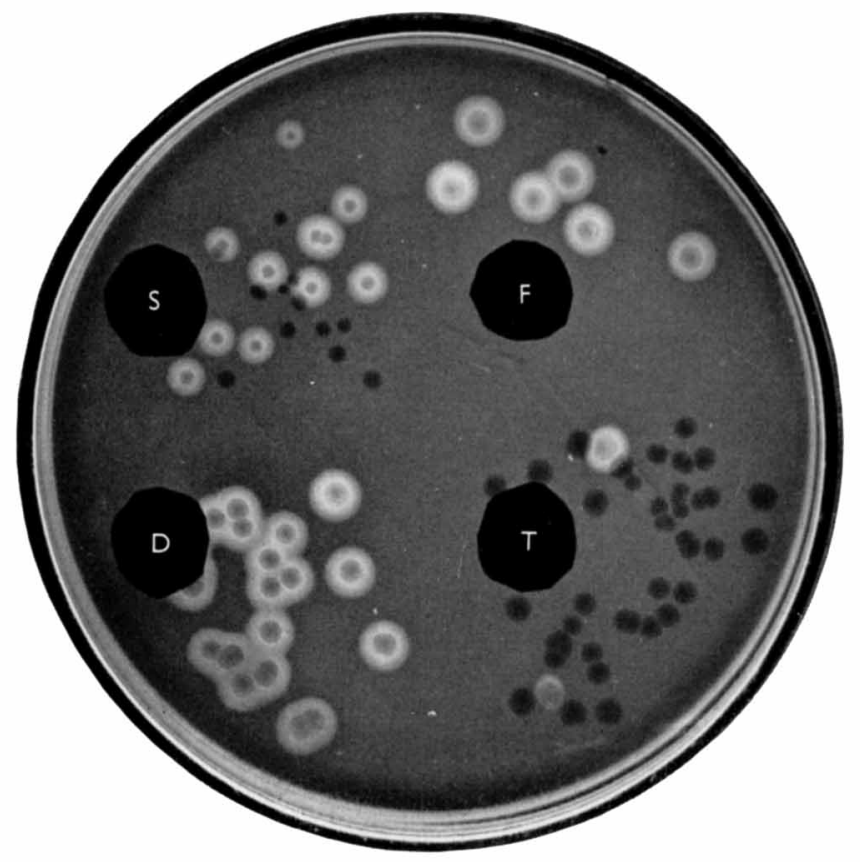

Fig. 2 


\section{The effect of acriflavine and ultraviolet radiation on the stability of Escherichia coli strains containing Hly factor}

Eight Hly+ strains of Escherichia coli, six wild and two laboratory-prepared, were grown at $37^{\circ}$ for $24 \mathrm{hr}$ in nutrient broth containing concentrations of acriflavine from those that had no apparent effect on growth to those which almost completely inhibited multiplication. When these cultures were inoculated on washed blood agar they yielded either no non-haemolytic colonies or, in the case of some of the cultures of two laboratory strains, only one or two, certainly no more than the number obtained from control cultures not containing acriflavine.

Suspensions of two of the strains were also ultraviolet-irradiated before being inoculated into broth containing acriflavine. Different exposure times were used, from one which caused no lethal effect to one which killed practically all the organisms. No non-haemolytic organisms were isolated.

\section{Immunity to reintroduction of Hly factor}

As mentioned previously, a very small number of non-haemolytic organisms were occasionally found during passage of four Hly+ strains of Escherichia coli. Attempt were made to reintroduce Hly factor into cultures of these non-haemolytic organisms. Five $\mathrm{Hly}^{+}$strains of $E$. coli were used as prospective donors. Three of these prospective donors were the parent strains from which three of the non-haemolytic segregants had been derived. Despite repeated attempts, it was impossible to reintroduce Hly factor into one of the four non-haemolytic cultures. The factor was reintroduced into the other three from only one of the five donors at a very low rate and then not at every attempt. By contrast, Hly factors were easily reintroduced into the non-haemolytic organisms obtained from $\mathrm{Hly}^{+}$Strains of Salmonella dublin and S. choleraesuis. Similar results were obtained with non-haemolytic organisms derived from the unstable $\mathrm{Hly}^{+}$strain of Shigella sonnei.

\section{The toxic effect in mice of haemolysin-containing culture fluids of strains of} Escherichia coli to which Hly factor had been transmitted in the laboratory

Mice injected intravenously with $0.6 \mathrm{ml}$. of haemolysin-containing bacteria-free fluids from cultures of nine strains of Escherichia coli to which Hly factor had been transferred in the laboratory became ill within $2 \mathrm{hr}$. They were dull, their respirations were more rapid and deeper and they had severe haemoglobinuria, signs similar to those previously observed in mice injected with culture fluids of $\alpha$-haemolytic strains of $E$. coli (Smith, 1963). Mice injected with similar amounts of culture fluid of the strains before Hly factor had been introduced into them showed little sign of ill-health.

The influence of Hly factor on the pathogenicity of Escherichia coli for mice

Hly factor did not appear to influence the pathogenicity for mice of Escherichia coli organisms given by the intraperitoneal route. For example, 3 groups of 15 mice were injected intraperitoneally with about $10^{8}$ viable organisms of either a non-haemolytic strain of $E$. coli $\mathrm{O} 18: \mathrm{K}$ ? suspended in phosphate buffer (pH 7.0), or of two cultures of this strain after the introduction of Hly factor from two serologically-different $\alpha$-haemolytic strains of $E$. coli. All three groups of mice became very ill and about one-third of those in each group died, all deaths occurring within $24 \mathrm{hr}$. 
The influence of Hly factor on the pathogenicity of salmonella for mice

Five mice were injected subcutaneously with 2000-3000 viable organisms of a $24 \mathrm{hr}$ broth culture of the non-haemolytic strain of Salmonella typhimurium referred to previously, and five mice with a similar dose prepared from this strain after the introduction of Hly factor. Identical experiments were made with the $S$. dublin and $S$. choleraesuis strains before and after the introduction of Hly factor. None of the 30 mice died. When they were killed 12 days after the beginning of the experiment there was no difference in the incidence and severity of the liver lesions in the mice which had been given haemolytic organisms and in those given non-haemolytic organisms.

In another experiment, 3 groups, each of 10 mice, were injected subcutaneously with one or other of the three haemolytic Salmonella strains. One mouse from each group was killed every day and its liver inoculated on to washed blood agar to determine the proportion of haemolytic and non-haemolytic salmonellas present. The highest ratio of haemolytic to non-haemolytic organisms of $S$. typhimurium found was 1:20; on several occasions only one or two haemolytic colonies amongst a mass of non-haemolytic colonies were seen on the washed blood agar plates. The haemolytic:non-haemolytic ratio found at all the daily examinations of the mice inoculated with haemolytic $S$. choleraesuis was about $1: 20$. In the first few examinations of the mice inoculated with $S$. dublin the ratio was $1: 2-5$; at subsequent examinations a progressive decrease in the relative numbers of haemolytic organisms was noted; at the final examination it was about 1:40.

\section{DISCUSSION}

The results indicate that the ability to produce $\alpha$-haemolysin can be transmitted from some strains of Escherichia coli and that transmission when it occurs is by conjugation. This is analogous to what has been found with $\mathrm{R}$ factors, col factors and F (Hayes, 1964; Datta, 1965). The evidence so far suggests that transmission may be considered to result from the presence of a supernumerary extrachromosomal genetic element, or plasmid, carrying a gene which determines the production of the haemolysin, in the same way as $\mathrm{R}$ factors carry the genes which confer drug resistance (Watanabe, 1963). The name 'Hly factor' is proposed for this element.

No exclusion of the kind described by Scaife \& Gross (1962) for F could be demonstrated between the Hly factor and either F or R factors; they could be carried together in the same bacterium. Nor could exclusion be demonstrated between Hly and col factors. When an $\mathrm{R}$ factor was present with Hly there was evidently no association between them, for they were transmitted independently of each other. In one $\mathrm{R}^{+}$ strain of $E$. coli, the particular $\mathrm{R}$ factor was apparently $f^{-}$(Watanabe et al. 1964) for $\mathrm{K} 12 \mathrm{~F}^{+}$strains into which it was introduced remained sensitive to the $\mathrm{F}$-specific phage. The Hly factor which this strain also contained, like the Hly factors in all the other strains tested, resembled the $f^{+} \mathrm{R}$ factors of Watanabe et al. (1964) in rendering $\mathrm{K} 12 \mathrm{~F}^{+}$strains resistant to the $\mathrm{F}$ specific phage.

The stability of Hly was high in most strains of Escherichia coli and Shigella and low in the Salmonella strains. Variability in stability is also a feature of $\mathbf{R}$ factors (Watanabe, 1963). Hly factor differed from $\mathrm{F}$ in not being eliminated by treatment 
with acriflavine or ultraviolet radiation, but many other plasmids, e.g. some $\mathbf{R}$ factors and col factors, are not eliminated in this way by either of these treatments.

Despite the fact that the haemolytic activity of all the strains of Escherichia coli examined appeared to be identical, it was not possible to demonstrate transmission from most of the strains. Several reasons may be put forward to account for this. Perhaps in these strains the genes responsible for haemolysin production are integrated in the chromosome. On the other hand, plasmids may be responsible, but ones which have never had or have lost conjugation-factor activity, or which are defective in this respect (Cuzin \& Jacob, 1965). Alternatively, the plasmids may simply be repressed like R factors (Meynell \& Datta, 1966); our techniques were such that low frequencies of transfer would not be detected.

Not only did the Hly + Salmonella and Escherichia coli strains differ greatly in stability but Hly was easily reintroduced into Salmonella organisms, but not into $E$. coli organisms, which had lost it. If the Hly system resembles the other systems previously referred to, then it probably consists of genes which govern the actual production of haemolysin and other genes responsible for the conjugation and transmission of these genes to other organisms. If this be so, it is possible that the Salmonella segregants had lost the complete genetic element whereas the $E$. coli organisms which lost the haemolytic activity still retained the part concerned with exclusion (Scaife \& Gross, 1962). It is possible that the few wild strains of $E$. coli into which we failed to introduce Hly excluded it for the same reason.

It would appear then that haemolytic activity is yet another transmissible character to add to the list of $\mathbf{R}$ factors, col factors and the antigen discovered by Ørskov \& Ørskov (1966). One might imagine that characters transmitted in this way should give some selective advantage to the organisms and it is easy to see, for example, what the advantage might be with $\mathrm{R}$ factors. The advantage in the present case might reflect pathogenicity, evidenced by experiments in which the culture fluids of strains of Escherichia coli before and after the introduction of Hly were injected intravenously into mice. Although no difference was observed in mice in experimental infections with whole organisms, the haemolysin itself was toxic. The great majority of strains of $E$. coli causing disease in pigs are $\alpha$-haemolytic.

We are grateful to Mr J. Snowden and Miss Susan Harding for their capable technical help. Our thanks are also due to Dr Naomi Datta, Mrs Esther Johnson, Dr Elinor Meynell, Dr K. C. Sellers and Mr J. R. Walton for help and assistance in various ways.

\section{REFERENCES}

CuZIN, F. \& JACOB, F. (1965). Analyse genétique fonctionnelle de l'épisome sexuel d'Escherichia coli. C.r. hebd. Séanc. Acad. Sci., Paris 260, 2087.

DatTA, N. (1965). Infectious drug resistance. Br. med. Bull. 21, 254.

Davis, J. E., Strauss, J. H. Jun. \& Sinsheimer, R. L. (1961). Bacteriophage MS2: another RNA phage. Science, N.Y. 134, 1427.

FredericQ, P. (1963). On the nature of colicinogenic factors; a review. J. theoret. Biol. 4, 159.

HAYES, W. (1964). The Genetics of Bacteria and their Viruses. Oxford: Blackwell Scientific Publications.

Meynell, E. \& DAtTA, N. (1966). The relation of resistance factors to the F-factor (sex factor) of Escherichia coli $\mathrm{K} 12$. Genet. Res. Camb. 7, 134.

ØRSKOV, I. \& ØRSKOV, F. (1966). Episome-carried surface antigen K88 of Escherichia coli. I. Transmission of the determinant of the K88 antigen and influence on the transfer of chromosomal markers. J. Bact. 91, 69. 
ScaIfe, J. \& Gross, J. D. (1962). Inhibition of multiplication of an F-Lac factor in Hfr cell of Escherichia coli K 12. Biochem. Biophys. Res. Commun. 7, 403.

Smith, H. Williams. (1963). The haemolysins of Escherichia coli. J. Path. Bact. 85, 197.

WATANABE, T. (1963). Infective heredity of multiple drug resistance in bacteria. Bact. Rev. $27,1$.

Watanabe, T., Nishida, H., Ogata, C., Arai, T. \& Sato, S. (1964). Episome-mediated transfer of drug resistance in Enterobacteriaceae. VII. Two types of naturally-occurring R factors. J. Bact. 88, 716.

\section{EXPLANATION OF PLATE \\ Plate 1}

Fig. 1. Result of a mixed culture experiment. The mixed culture of an $\alpha$-haemolytic donor strain of Escherichia coli and a non-haemolytic recipient strain of $E$. coli has been inoculated on to a washed blood agar plate containing an antibiotic which has completely suppressed the growth of the donor strain. The colonies are those of the antibiotic-resistant recipient strain; about $10 \%$ of them are now haemolytic. $\times 1 \frac{1}{2}$.

Fig. 2. Strains of shigellas and salmonellas into which Hly factor has been introduced. The plate has been lightly inoculated with suspensions of haemolytic colonies of Shigella sonnei $(\mathbf{S})$, S. flexneri $(\mathrm{F})$, Salmonella typhimurium (T) and S. dublin (D). The instability of the Hly factor in Shigella sonnei and Salmonella typhimurium strains is apparent. $\times 1 \frac{1}{3}$. 